\title{
AN ISOPERIMETRIC INEQUALITY FOR CLOSED CURVES CONVEX IN EVEN-DIMENSIONAL EUCLIDEAN SPACES*
}

BY

\author{
I. J. SCHOENBERG \\ in Philadelphia, Pa.
}

\section{Introduction}

1. The main theorem. A closed convex curve in the plane $E_{2}$ is usually defined as the boundary of a compact convex set. ${ }^{1}$ Alternatively, if the curve is given in parametric form we could say that the curve is convex provided it never crosses a straight line more than twice. The second definition has the advantage of extending in a natural way to closed curves in an even-dimensional space $E_{2} n$ as follows:

Let

$$
C: \quad x_{i}=x_{i}(t), \quad(i=1, \ldots, 2 n ; 0 \leq t=2 \pi),
$$

where $x_{i}(t)$ are continuous functions of period $2 \pi$, be a closed curve in $E_{2 n}$. We shall say that $C$ is convex in $E_{2 n}$ provided that it never crosses a hyperplane more than $2 n$ times. If $C$ is convex in $E_{2 n}$ and spans the space $E_{2 n}$, i.e. is not contained in a lower-dimensional flat space, then we shall say that $\left(\because\right.$ is convex on $E_{2} n$. It will be shown below (Article 5) that curves convex in $E_{2 n}$ are rectifiable. As an example of a curve convex on $E_{2 n}$ we mention the curve

$$
\begin{aligned}
C_{0}: \quad x_{1} & =\cos t, x_{3}=\frac{1}{2} \cos 2 t, \ldots, x_{2 n-1}=\frac{1}{n} \cos n t, \\
x_{2} & =\sin t, x_{4}=\frac{1}{2} \sin 2 t, \ldots, x_{2 n}=\frac{1}{n} \sin n t, \quad(0 \leq t \leq 2 \pi) .
\end{aligned}
$$

Indeed, $C_{0}$ is convex in $E_{2 n}$, for if $l\left(x_{1}, \ldots, x_{2 n}\right)$ is any linear function and if we substitute the $x_{i}$ as defined by (2), we find that $l=T_{n}(t)$ is a real trigonometric

* This work was performed on a National Bureau of Standards contract with the University of California, Los Angeles, and was sponsored (in part) by the Office of Scientific Research, USAF.

1 See [1], page 3, in the list of references at the end of this paper.

10-533807. Acta mathematica. 91. Imprimé lo 27 octobre 1954. 
polynomial of order $n$ which is known never to change sign more than $2 n$ times within a period.

The main result is the following theorem

I. Let $C$ be a closed curve convex in $E_{2 n}$ and let $L$ denote its length. Let $K=K(C)$ be the convex hull of $C$ and let $V=V(K)$ denote the 2 -dimensional volume of $K$. Then the following inequality holds

$$
L^{2 n} \geq(2 \pi n)^{n} n !(2 n) ! V(K)
$$

with the equality sign if and only if the curve $C$ agrees up to a rigid motion and a similitude, followed perhaps by a reflexion, with the curve $C_{0}^{2}$ defined by (2).

If $n=1$, (3) reduces to the classical isoperimetric inequality $L^{2} \geq 4 \pi V$ for curves convex in the plane, $V$ now denoting the area enclosed by $C$, and where equality holds only if $C$ is a circle. The inequality (3) shows that among all closed curves convex in $E_{2 n}$ and of given length $L$, only those which are similar to $C_{0}$ will maximize the volume $V(K(C))$ of their convex hull.

2. Two related theorems. Theorem I will follow from the following theorem in which there is no reference to convex curves:

II. Let $x_{t}(t),(i=1, \ldots, 2 n)$, be absolutely continuous functions of period $2 \pi$, not all being constant and such that $x_{i}^{\prime}(t) \in L_{2}(0,2 \pi)$. Then the following inequality holds

(4) $\left\{\int_{0}^{2 \pi}\left(\sum_{1}^{2 n} x_{i}^{\prime 2}\right) d t\right\}^{n} \geq n^{n} \int_{0}^{2 \pi} \ldots \int_{0}^{2 \pi} \operatorname{det}\left\|x_{i}\left(t_{1}\right), x_{i}^{\prime}\left(t_{1}\right), x_{i}\left(t_{2}\right), x_{i}^{\prime}\left(t_{2}\right), \ldots, x_{i}\left(t_{n}\right), x_{i}^{\prime}\left(t_{n}\right)\right\| d t_{1} \ldots d t_{n}$, the integrand of the right-hand side being a determinant of order $2 n$ whose ith row is written out. Moreover, we have the equality sign if and only if the functions $x_{i}(t)$ arise from the special set of functions (2) by a right-handed orthogonal transformation followed by a similitude with positive ratio and a translation.

This theorem will be established by means of Fourier series, a method first used by $A$. Hurwitz ${ }^{3}$ for the special case when $n=1$. In this classical case, Fourier expansions reduce the problem to a quadratic inequality easily established by completing squares. It will be seen by means of Fourier expansions that Theorem II is equivalent to the following theorem

The curve $C_{0}$ is a simple example of a closed gcrew-line in $E_{2 n}$ (see [10]). A curve $C_{1}$, related to $C_{0}$ by similitudes, and the corresponding $K\left(C_{1}\right)$, play an important role in Caratritodory's paper [2] and also in the so-called trigonometric moment problem.

See [8]. 
III. Let

$$
M=\left\|a_{i 1}, b_{i 1}, a_{i 2}, b_{i 2}, \ldots, a_{i m}, b_{i m}, \ldots\right\|, \quad(i=1, \ldots, 2 n),
$$

be a real matrix of $2 n$ rows and infinitely many columns, $M \neq 0$, and such that the sum of the squares of the elements in every row converges. Let

Setting

$$
\begin{gathered}
D\left(j_{1}, j_{2}, \ldots, j_{n}\right)=\operatorname{det}\left\|a_{i j_{1}}, b_{i j_{1}}, a_{i j_{2}}, b_{i j_{2}}, \ldots, a_{i j_{n}}, b_{i j_{n}}\right\|, \\
\left(1 \leq j_{1}<j_{2}<\cdots<j_{n}\right) .
\end{gathered}
$$

and

$$
S=\sum_{v=1}^{\infty} \sum_{i=1}^{2 n}\left(a_{i v}^{2}+b_{i v}^{2}\right)
$$

$$
\Phi=\sum_{j_{1}<\ldots<j_{n}} \frac{1}{j_{1} j_{2} \ldots j_{n}} D\left(j_{1}, j_{2}, \ldots, j_{n}\right)
$$

then the last series converges absolutely and we have the inequality

$$
S^{n} \geq(2 n)^{n} n ! \Phi
$$

with equality holding if and only if the matrix (5) has only zero elements, except in its first $2 n$ columns which form a square matrix with elements positively proportional to a right-orthogonal matrix.

The section-headings describe the contents of the paper. The discussion starts with an account of indispensable properties of convex polygons and curves.

\section{\$ 1. On closed curves convex in $E_{2 n}$ and the volumes of their convex hulls}

3. On convex polygons. Let

$$
\Pi=P_{0} P_{1} \ldots P_{k}, P_{i}=\left(x_{i 1}, x_{i 2}, \ldots, x_{i m}\right)
$$

be a polygon with vertices in $E_{m}$. We assume that $\Pi$ spans $E_{m}$, which is the case provided the matrix

$$
X=\left\|1, x_{i 1}, x_{i 2}, \ldots, x_{i m}\right\|, \quad(i=0, \ldots, k ; k \geq m),
$$

is of rank $m+1$. We introduce the following

Definition 1. We say that the polygon $\Pi$ is convex on $E_{m}$ provided it spans $E_{m}$ and crosses no hyperplane more than $m$ times. If in this definition we do not require that $\Pi$ should span $E_{m}$, then we say that $\Pi$ is convex in $E_{m}$.

The convexity of $\Pi$ in (or on) $E_{m}$ evidently means the following: If $l(P)=$ $=l\left(x_{1}, \ldots, x_{m}\right)$ is an arbitrary linear function of the coordinates, then in the sequence 
of numbers $l\left(P_{0}\right), l\left(P_{1}\right), \ldots, l\left(P_{k}\right)$, we should have at most $m$ changes of signs. Now the important difference between "convexity in $E_{m}$ " and "convexity on $E_{m}$ " becomes apparent. Indeed, for "convexity on $E_{m}$ " the following lemma is identical, except in terminology, to a theorem of F. Gantmakher and M. Krein ${ }^{4}$ :

Lemma 1. The polygon $\Pi$ is convex on $E_{m}$ if and only if the matrix $X$, defined by (1.2) is of rank $m+1$ and all its non-vanishing minors of order $m+1$ are of the same sign.

This lemma seems intuitively clear for the case of the plane $E_{2}$, for it says that the convexity of $\Pi$ on $E_{2}$ requires that no two among the triangles $P_{\alpha} P_{\beta} P_{\gamma}(\alpha<\beta<\gamma)$ should have opposite orientations.

We are now confronted with a basic distinction depending on the parity of the dimension number $m$. If $m$ is even we readily see that if $\Pi$ is convex on $\boldsymbol{E}_{m}$, then also the closed polygon

$$
\mathrm{II}_{1}=P_{0} P_{1} \ldots P_{k} P_{0}
$$

is convex on $E_{m}$. Indeed, if $\pi(l(\mathrm{P})=0)$ is a hyperplane and II crosses $\pi$ less than $m$ times, then also $\mathrm{II}_{1}$ will not cross $\pi$ more than $m$ times. However, if $\Pi$ crosses $\pi$ exactly $m$ times, then $P_{0}$ and $P_{k}$ are never on opposite sides of $\pi$ ( $m$ being even), so that the last side $P_{k} P_{0}$ does not cross $\pi$. For the closed polygon $I_{1}$ it does not matter which vertex is taken to be the first, as long as the correct cyclic order of the vertices is preserved. The convexity of $I I_{1}$ may also be described in a way which ignores ab initio which vertex might be the first: $1 \mathrm{I}_{1}$ should never cross $\pi$ more than $m$ times, as we go once around the polygon. Notice that the number of such crossings is always even. The same indifference to cyclic permutations is also shared by the criterion of Lemma 1: Cyclic permutations of the rows of the matrix $X$ will not change the common sign of its minors of the odd order $m+1$.

The situation is quite different if $m$ is odd. In this case it can be shown that the first and the last vertex of a polygon, convex on $E_{m}$, can never coincide.

4. On convex curves. The above definitions and results extend readily to con. tinuous arcs and closed curves. In view of our particular aim we restrict the discussion to even dimensions $m=2 n$ and closed curves. The basic definition is as follows:

4 See their recent book [4], Theorem 3, page 297. Also proved in [14], Theorem 1. AnNe Whitney and the author were unaware of the book by Gantmakrer and Krein when [14] was published. However, the priority clearly belongs to the Russian authors. See also footnote 5 for the connection with the work of J. HJELMSLEv. 
Definition 2. The closed curve

$$
C: \quad x_{i}=x_{i}(t) \quad(i=1, \ldots, 2 n ; 0 \leq t \leq 2 \pi)
$$

where the continuous functions $x_{i}(t)$, defined for all $t$, have the period $2 \pi$, is said to be convex on $E_{2 n}$ provided $C$ spans $E_{2 n}$ and all closed polygons inscribed in $C$ are convex in $E_{2 n}$. If in this definition we do not require that $C$ should span $E_{2 n}$, then we say that $C$ is convex in $E_{2 n} .^{5}$

A criterion is given by

Lemma 2. The closed curve $C$, defined by (1.3), is convex on $E_{2 n}$ if and only if the determinants

$$
\Delta=\operatorname{det}\left\|1, x_{1}\left(t_{i}\right), \ldots, x_{2 n}\left(t_{i}\right)\right\|\left(t_{0}<t_{1}<\cdots<t_{2 n}<t_{0}+2 \pi\right)
$$

do not all vanish and the non-vanishing ones have the same sign.

Proof. Let us inscribe in $C$ a closed polygon $\Pi=P_{0} P_{1} \ldots P_{k} P_{0}$ corresponding to the parameter values

$$
t_{0}<t_{1}<\cdots<t_{k}<t_{0}+2 \pi
$$

The conditions are necessary. Assume $C$ convex on $E_{2 n}$. Let II span $E_{2 n}$. But then, by Lemma 1, the matrix

$$
X=\left\|1, x_{1}\left(t_{i}\right), \ldots, x_{2 n}\left(t_{i}\right)\right\| \quad(i=0,1, \ldots, k)
$$

s Professor W. Fenchel kindly called to my attention the fundamental paper [7] of Hueluslev. Huelmslev ([7], pp. 4, 6, 7, 23, 41) calls the polygon II, of (1.1), monotone. provided the matrix (1.2) satisfies the conditions of Lemma 1 . By Lemma 1 we thus see that $I$ is convex on $E_{m}$ if and only if $\mathrm{II}$ is monotone in the sense of HJELMSLEv. Similarly, in view of our Definition 2 and Lemma 2 we see that the curve (1.3) is convex on $E_{2 n}$ if and only if it is monotone in the sense of HJELMSLEv ([7], p. 60). We should also point out the connection with the concept of an arc of order $m$ in $E_{m}$ due to C. Juel; see Marchaud [9] and Scherk [13], also for references. Arcs of order $m$ in $E_{m}$ are convex on $E_{m}$ but not conversely. A theorem of HJELMSLEv ([7], p. 62) should be especially quoted, being closely related to our Lemma 2 . Its statement for the closed curve (1.3) in $E_{2} n$ is as follows: If no subarc of $C$ is in a $E_{2 n-1}$, then $C$ is of order $2 n$ if and only if all determinants (1.4) are posiuive or all are negative. One may even omit in the statement the assumption concerning the subarcs of $C$. In [15] I have recently said (p. 227) that by this theorem HJELMSLEv has essentially anticipated the result of Gantmakher-Krein. This view was perhaps exaggerated.

$I$ also owe to W. Fenchel the following reference: E. Egervary, On the smallest convex cover of a simple arc of space-curve, Publ. math. Debrecen, $1(1949), 65-70$, in which its author solves the problem of the present paper for open arcs in $E_{3}$. As yet unsolved is the problem for the particularly interesting case of closed curves in $E_{3}$ (see [1], footnote on p. 111). 
has all non-vanishing minors of order $2 n+1$ of the same sign. Given two non. vanishing determinants $\Delta_{1}, \Delta_{2}$, of the form (1.4), we may so choose the parameter values (1.5), that $\Delta_{1}$ and $\Delta_{2}$ are among the minors of $X$. But then $\Delta_{1} \Delta_{2}>0$.

The conditions are sufficient. This follows directly by Lemma 1 and Definitions 1 and 2.

Remarks. 1. Our old definition of a closed polygon convex on $E_{2 n}$ agrees with the new definition if applied to the polygon considered as a closed curve.

2. Lemma 2 may be rephrased in geometric terms as follows: The continuous closed curve $P=P(t)$ in $E_{2 n}$ is convex on $E_{2 n}$ if and only if it has the following properties: If $P_{0}, P_{1}, \ldots, P_{2 n}$ are $2 n+1$ points on $C$, in correct cyclic order corresponding to increasing values of $t$, then the $2 n$-dimensional simplices $\left[P_{0}, P_{1}, \ldots, P_{2 n}\right]$ should not be all degenerate and the non-degenerate ones should have the same orientation.

3. It seems natural and useful to classify the curves convex on $E_{2 n}$ into positively convex and negatively convex curves, depending on the common sign of the determinants $\Delta$, or the common orientation of the simplices $\left[P_{0}, P_{1}, \ldots, P_{2 n}\right]$.

4. An adaptation of these definitions and results to open arcs in even or odd. dimensional spaces seems perfectly straight-forward. As an example we mention that one full turn (but no longer arc) of a right-handed circular helix in $E_{3}$ is positively convex on $E_{3} \cdot{ }^{\circ}$

5. Lemma 2 applies easily to show that the curve $C_{0}$, defined by (2), is positively convex on $E_{2 n}$. Indeed, in this case the determinant (1.4), familiar from the problem of trigonometric interpolation, may be evaluated explicitly and we find

$$
\Delta=\frac{2^{2 n^{\prime}}}{n !} \prod_{\mu>v} \sin \frac{t_{\mu}-t_{v}}{2}>0 .
$$

5. Convex curves are rectiflable. ${ }^{7}$ We need the following

Definition 3. The bounded real function $f(t), a \leq t \leq b$, is said to be non-oscillatory provided there is a fixed natural integer $N$ such that for every real $c$ the function $f(t)-c$ changes sign at most $N$ times in the range $[a, b]$.

With this definition we have

- In this example which can be widely generalized we are especially close to PóLYA's paper [12].

? The contents of Article 5 where developed in the course of a conversation with TH. S. MotzkrN. Here we are close to Marchaud's work [9], Chapitre I. 
Lemma 3. If $f(t)$ is non-oscillatory in the range $[a, b]$, then $f(t)$ is of bounded variation, in fact

$$
\text { Total variation of } f(t) \leq N(\sup f-\inf f)
$$

Proof. Let $a_{0}=t_{0}<t_{1}<\cdots<t_{k}=b$ and let $P_{i}=\left(t_{i}, f\left(t_{i}\right)\right)$ be the corresponding points on the graph $x=f(t)$. Consider the polygon $P_{0} P_{1} \ldots P_{k}$ and its orthogonal projection $P_{0}^{\prime} P_{1}^{\prime} \ldots P_{k}^{\prime}$ into the segment $I=[\inf f \leq x \leq \sup f]$ of the $x$-axis. Since $P_{0} P_{1} \ldots P_{k}$ may cross a horizontal line $x=c$ at most $N$ times we conclude that the $k$ open (or void) segments $P_{0}^{\prime} P_{1}^{\prime}, P_{1}^{\prime} P_{2}^{\prime}, \ldots, P_{k-1}^{\prime} P_{k}^{\prime}$ may cover a point of $I$ at most $N$ times. But then

or

$$
\sum_{\nu=0}^{k-1} P_{\nu}^{\prime} P_{\nu+1}^{\prime} \leq N \cdot m(I)
$$

$$
\sum_{v=0}^{k-1}\left|f\left(t_{v+1}\right)-f\left(t_{v}\right)\right| \leq N(\sup f-\inf f)
$$

whence (1.6) follows on taking the supremum of the left side.

This result clearly implies

Lemma 4. A closed curve $C$ convex in $E_{2 n}$ is necessarily rectifiable.

Indeed, let $C$ be defined by the equations (1.3), say. Now $f(t)=x_{i}(t)$ being continuous is also bounded in the range $[0,2 \pi]$. However, this function is also nonoscillatory, with $N=2 n$ in Definition 3 , because $C$ may cross the hyperplane $x_{i}=c$ at most $2 n$ times. By Lemma 3 all $x_{i}(t)$ are of bounded variation, which proves Lemma 4.

6. On the volume of polyhedra spanned by convex polygons. Let II be a closed polygon convex on $E_{2 n}$. We wish to express the volume of the convex polyhedron $K(I I)$ in terms of volumes of simplices. We restrict our discussion to the first significant case when $n=2$. The simplification in notations thus afforded is considerable, it being obvious at all times how the general case is to be treated.

Lemma 5. Let $\Pi=P_{0} P_{1} \ldots P_{m} P_{0}$ be a closed polygon in $E_{4}$ which is convex on $E_{4}$. Let $K=K(\Pi)$ denote its convex hull, $V(K)$ the volume of $K$. Let $O$ be a point in the interior of $K$. The volume of $K$ is given by the formula

$$
V(K)=\frac{1}{2 !} \sum_{i, j=0}^{m} V\left[O, P_{i}, P_{i+1}, P_{j}, P_{j+1}\right], \quad\left(P_{m+1}=P_{0}\right)
$$

where the summand is the volume of the simplex of vertices $\Theta, P_{i}, P_{i+1}, P_{j}, P_{j+1}$, it being zero if the simplex is degenerate. 
Proof. (i) By Lemma 1 we know that the matrix

$$
X=\left\|1, x_{i 1}, x_{i 2}, x_{i 3}, x_{i 4}\right\|, \quad(i=0,1, \ldots, m),
$$

is of rank 5 and that all non-vanishing minors of order 5 are of the same sign. There is no restriction in assuming these minors to be positive, i.e. the polygon $\Pi$ to be positively convex on $E_{4}$. However, we shall assume for the moment that

$$
\text { All 5th order minors of } X \text { are positive, }
$$

showing later how this restriction can be removed.

To abbreviate our notation let

$$
D\left(x, x_{i}, x_{j}, x_{k}, x_{l}\right)=\left|\begin{array}{ccccc}
1 & x_{1} & x_{2} & x_{3} & x_{4} \\
1 & x_{i 1} & x_{i 2} & x_{i 3} & x_{i 4} \\
1 & x_{j 1} & x_{j 2} & x_{j 3} & x_{j 4} \\
1 & x_{k 1} & x_{k 2} & x_{k 3} & x_{k 4} \\
1 & x_{l 1} & x_{l 2} & x_{l 3} & x_{l 4}
\end{array}\right| .
$$

The assumption (1.9) implies that every four points $P_{l}, P_{j}, P_{k}, P_{l}(0 \leq i<j<k<l \leq m)$ determine uniquely a 3 -flat $\pi(i, j, k, l)$, of equation $D\left(x, x_{i}, x_{j}, x_{k}, x_{l}\right)=0$, containing no other point $P_{v}$ since $D\left(x_{v}, x_{i}, x_{j}, x_{k}, x_{l}\right) \neq 0$ if $\nu \neq i, j, k, l$. We now assert the following: All 3-dimensional faces of $K(\mathrm{II})$ are precisely in the 3-flats

$$
\begin{array}{ll}
\pi(i, i+1, j, j+1), & (0 \leq i<i+1<j<j+1 \leq m), \\
\pi(0, i, i+1, m), & (0<i<i+1<m) .
\end{array}
$$

Indeel, that these 3 -flats contain 3 -faces of $K(I I)$ is seen as follows: The assumption (1.9) implies the inequalities

$$
\begin{aligned}
& D\left(x_{v}, x_{i}, x_{i+1}, x_{j}, x_{j+1}\right)>0 \quad \text { if } \quad v \neq i, i+1, j, j+1, \\
& D\left(x_{v}, x_{0}, x_{i}, x_{i+1}, x_{m}\right)<0 \quad \text { if } \quad v \neq 0, i, i+1, m,
\end{aligned}
$$

which show that each of the planes (1.10) leave all the other vertices strictly on one side. The 3 -flats (1.10) are therefore planes of support of $K(\Pi)$. On the other hand none of the 3-flats through four of the points, other than the 3-flats (1.10), can possibly be a plane of support of $K(\Pi)$, for such a 3 -flat is seen to have some of the vertices on one of its sides and others on the other side. We conclude ${ }^{8}$ that

8 From this result and its extension to $E_{2 n}$ it is easy to derive the following: The curve $C$ convex on $E_{2 n}$ is on the boundary of its convex hull $K(C)$. This is closely related to a result of W. Gustin [5]. 
the 3 -dimensional faces of $K(I)$ are the simplices $\left[P_{i}, P_{i+1}, P_{j}, P_{j+1}\right]$, where the pair $(i, j)$ runs over the set $S$ of pairs $(i, j)$ defined by the conditions

$$
0 \leq i<i+1<j<j+1 \leq m \text { or } 0<i<i+1<j=m .
$$

We may summarize the situation by the relation

$$
\text { Boundary of } K(\Pi)=\sum_{S}\left[P_{i}, P_{i+1}, P_{j}, P_{j+1}\right], \quad\left(P_{m+1}=P_{0}\right) \text {. }
$$

If $O$ is a point interior to $K=K(\Pi)$ we obtain, by central projection from $O$, the relation

$$
K=\sum_{S}\left[O, P_{i}, P_{i+1}, P_{j}, P_{j+1}\right]
$$

which describes a dissection of $K$ into 4-dimensional simplices. On passing to volumes we obtain

$$
V(K)=\sum_{S} V\left[O, P_{i}, P_{i+1}, P_{j}, P_{j+1}\right]
$$

Let us observe now that all simplices on the right-hand side of (1.12) are positive y oriented. Indeed, by (1.11) we see that if $(i, j) \in S$ then all determinants $D\left(x_{y}, x_{i}\right.$, $\left.x_{i+1}, x_{j}, x_{j+1}\right)(v \neq i, i+1, j, j+1)$ are positive. But then also $D\left(x, x_{i}, x_{i+1}, x_{j}, x_{j+1}\right)>0$ if the point $(x)$ is in the interior of $K$, this determinant being a linear combination with positive coefficients of non-negative determinants some of which must be positive. On choosing the point $O$ as the origin of the coordinate system we have

$$
V(K)=\sum_{S} V\left[O, P_{i}, P_{i+1}, P_{j}, P_{j+1}\right]=\sum_{4}^{1} \sum_{S} D\left(0, x_{i}, x_{i+1}, x_{j}, x_{j+1}\right) .
$$

Finally, the last expression may be written as

$$
V(K)=\frac{1}{2 ! 4 !} \sum_{i, j=0}^{m} \operatorname{det}\left\|x_{i v}, x_{i+1, \nu}, x_{j v}, x_{j+1, \nu}\right\|,
$$

since all new terms entering into the double summation will vanish if they have coincident rows while the duplication of old terms is offset by the new factor 2 ! in the denominator. Now (1.13) is precisely the relation (1.7) we wished to establish.

(ii) We now wish to remove the assumption (1.9) on which we relied heavily in our previous discussion. We do this by the following simple device. We need the matrix

$$
\left\|q^{(i-j)^{1}}\right\|_{i, j=0, \ldots, m}, \quad(0<q<1) .
$$

It follows from a theorem of Pólya ${ }^{9}$ that all minors of this matrix, of all orders, are positive. Moreover, this matrix clearly converges to the unit matrix as $q \rightarrow 0$.

${ }^{9}$ See [11], Problem 76, page 49. 
Dividing the elements of each row by the sum of all elements in that row we obtain a new matrix which we denote by $H_{q}$. Returning to the matrix (1.8) we form the product

$$
X^{*}=H_{q} X=\left\|1, x_{i 1}^{*}, x_{i 2}^{*}, x_{i 3}^{*}, x_{i 4}^{*}\right\|_{i=0, \ldots, m}
$$

and easily infer the following: The new matrix $X^{*}$, whose elements depend on $q$, enjoys the property (1.9) and defines a closed polygon

$$
\Pi^{*}=P_{0}^{*} P_{1}^{*} \ldots P_{m}^{*} P_{0}^{*} \text { in } E_{4},
$$

whose vertices are as close to those of $\Pi$ as we wish, provided $q$ is sufficiently small. Iet $K^{*}=K\left(\Pi^{*}\right)$. By our previous discussion we know that $V\left(K^{*}\right)$ may be expressed by the analogue of (1.13). Also $V\left(K^{*}\right) \rightarrow V(K)$, as $q \rightarrow 0$. By continuity we see that (1.13) again holds even if the condition (1.9) is disregarded.

7. The volume $V(K(C))$ expressed as an integral. We are now turning to the main result of this section:

Theorem 1. Let $C$ be a closed curve convex on $E_{2 n}$ defined by

$$
C: \quad x_{i}=x_{i}(t), \quad(i=1, \ldots, 2 n ; 0 \leq t \leq 2 \pi) .
$$

We assume the $x_{i}(t)$ to be absolutely continuous in $[0,2 \pi]$, a condition which is auto. matically fulfilled if the parameter $t$ is proportional to the arc-length along $C$. Then the volume of the convex hull $K(C)$ may be expressed by the following Lebesgue integral

$$
V(K(C))=\frac{\varepsilon}{n !(2 n) !} \int_{0}^{2 \pi} \ldots \int_{0}^{2 \pi} \operatorname{det}\left\|x_{i}\left(t_{1}\right), x_{i}^{\prime}\left(t_{1}\right), \ldots, x_{i}\left(t_{n}\right), x_{i}^{\prime}\left(t_{n}\right)\right\| d t_{1} \ldots d t_{n},
$$

where $\varepsilon=+1$ or -1 depending on whether $C$ is positively or negatively convex on $E_{2 n}{ }^{10}$

Proof. Again, to simplify our notation we assume that $n=2$. Let us assume that $C$ is positively convex on $E_{4}$. Divide the range $[0,2 \pi]$ in $2^{k}$ equal parts by the points

$$
t_{v}=2 \pi v / 2^{k}, \quad\left(v=0,1, \ldots, m=2^{k}-1\right),
$$

and let $P_{v}$ be the corresponding point on $C$. The inscribed polygon $\Pi=P_{0} P_{1} \ldots P_{m} P_{0}$ being positively convex on $E_{4}$, provided $k$ is sufficiently large, by (1.13) we have

$$
V(K(\Pi))=\frac{1}{2 ! 4 !} \sum_{i, j=0}^{m} \operatorname{det}\left\|x_{v}\left(t_{i}\right), x_{v}\left(t_{i+1}\right), x_{v}\left(t_{j}\right), x_{v}\left(t_{j+1}\right)\right\| .
$$

10 We lose no generality by restricting our discussion to curves convex on $E_{2 n}$, for if $C$ is contained in a lower-dimensional flat space, then both sides of (1.15) evidently vanish. 
Subtracting the first and third column of the determinant from those just ahead we obtain

$$
V(K(\Pi))=\frac{1}{2 ! 4 !} \sum_{i, j=0}^{m} \operatorname{det}\left\|x_{v}\left(t_{i}\right), \int_{t_{i}}^{t_{i+1}} x_{v}^{\prime}\left(\tau_{1}\right) d \tau_{1}, x_{\nu}\left(t_{j}\right), \int_{t_{j}}^{t_{j+1}} x_{\nu}^{\prime}\left(\tau_{2}\right) d \tau_{2}\right\|
$$

and finally

$$
V(K(\Pi))=\frac{1}{2 ! 4 !} \sum_{i, j \rightarrow 0}^{m} \int_{t_{i}}^{t_{i+1}} \int_{t_{j}}^{t_{j+1}} \operatorname{det}\left\|x_{v}\left(t_{i}\right), x_{v}^{\prime}\left(\tau_{1}\right), x_{v}\left(t_{j}\right), x_{\nu}^{\prime}\left(\tau_{2}\right)\right\| d \tau_{1} d \tau_{2} .
$$

A passage to the limit in this relation, as $k \rightarrow \infty$, presents no difficulties. On the left side $V(K(\Pi)) \rightarrow V(K(C))$. Indeed, the inclusion $K(\Pi) \subset K(C)$ implies that

$$
V(K(I)) \leq V(K(C)) .
$$

On the other hand the increasing sequence of sets $K(\Pi)$ converges to a limit which includes the set $K_{0}(C)$ of interior points of $K(C)$. This remark and (1.17) imply

$$
\text { measure of } K_{0}(C) \leq \lim _{k \rightarrow \infty} V(K(\mathrm{II})) \leq V(K(C))
$$

and the equality of the extreme terms implies the desired conclusion.

There remains to show that the sum $S$ on the right-hand side of (1.16) converges to a double integral which we may write as

$$
J=\sum_{i, j=0}^{m} \int_{t_{i}}^{t_{i}+1} \int_{i_{j}}^{t_{j+1}} \operatorname{det}\left\|x_{v}\left(\tau_{1}\right), x_{v}^{\prime}\left(\tau_{1}\right), x_{v}\left(\tau_{2}\right), x_{v}^{\prime}\left(\tau_{2}\right)\right\| d \tau_{1} d \tau_{2} .
$$

In order to show that $S \rightarrow J$, we expand each of the two determinants into 4 ! terms and find for their difference the expression

$$
\sum \pm x_{v_{2}}^{\prime}\left(\tau_{1}\right) x_{v_{1}}^{\prime}\left(\tau_{2}\right)\left[x_{v_{1}}\left(t_{i}\right) x_{v_{2}}\left(t_{j}\right)-x_{v_{2}}\left(\tau_{1}\right) x_{v_{3}}\left(\tau_{2}\right)\right]
$$

Given $\varepsilon>0$, the equi-uniform continuity of all products $x_{r}\left(\tau_{1}\right) x_{s}\left(\tau_{2}\right)$ shows that all square brackets will be in absolute value less than $\varepsilon$, each within its respective cell

$$
t_{i} \leq \tau_{1} \leq t_{i+1}, \quad t_{j} \leq \tau_{2} \leq t_{j+1},
$$

provided that $k$ is sufficiently large. But then

$$
|S-J| \leq 2 \varepsilon \sum \int_{0}^{2 \pi} \int_{0}^{2 \pi}\left|x_{\nu_{z}}^{\prime}\left(\tau_{1}\right)\right| \cdot\left|x_{\nu_{1}}^{\prime}\left(\tau_{2}\right)\right| d \tau_{1} d \tau_{2}=A \cdot \varepsilon
$$


This concludes a proof of Theorem 1 for $n=2$. The alterations necessary to deal with the general case of $E_{2 n}$ are deemed to be obvious in every respect.

8. A proof that Theorem II implies the isoperimetric inequality of Theorem I. Let the curve $C$, defined by (1), be positively convex on $E_{2 n}$. Let us assume, moreover, that the parameter $t=2 \pi s / L$, where $s$ is the arc length along $C$. For these functions $x_{i}(t)$ we have the inequality (4) of Theorem II which we take for granted for the moment. Both sides of (4) have a geometric meaning which we wish to derive. By (1.15), the right-hand side of $(4)$ is equal to

Since

$$
n^{n} n !(2 n) ! V(K(C))
$$

$$
\sum_{1}^{2 n} x_{i}^{\prime 2}=\left(\frac{d s}{d t}\right)^{2}=\frac{L^{2}}{4 \pi^{2}}
$$

holds almost everywhere, the left-hand side of (4) equals

$$
\left(\int_{0}^{2 \pi} \frac{L^{2}}{4 \pi^{2}} d t\right)^{n}=(2 \pi)^{n} L^{2 n}
$$

Thus (4) reduces to the isoperimetric inequality (3) with equality only if $C$ is similar to $C_{0}$.

\section{\$ 2. Further properties of $V(K(C))$ and reformulation of Theorem II in terms of Fourier series}

9. An expression for $V(K(C))$ in terms of areas of 2-dimensional projections of $C$. Assuming the curve $C$ to be positively convex on $E_{2 n}$, we have found the expression

$$
V(K(C))=\frac{1}{n !(2 n) !} \int_{0}^{2 \pi} \ldots \int_{0}^{2 \pi} \operatorname{det}\left\|x_{i}\left(t_{1}\right), x_{i}^{\prime}\left(t_{1}\right), \ldots, x_{i}\left(t_{n}\right), x_{i}^{\prime}\left(t_{n}\right)\right\| d t_{1} \ldots d t_{n}
$$

We now expand the determinant, by a repeated application of Laplace's rule, by second-order minors formed from the pairs of columns $(1,2),(3,4), \ldots,(2 n-1,2 n)$, obtaining a sum

$$
\sum \pm\left|\begin{array}{ll}
x_{\mu_{1}}\left(t_{1}\right) & x_{\mu_{1}}^{\prime}\left(t_{1}\right) \\
x_{v_{1}}\left(t_{1}\right) & x_{v_{1}}^{\prime}\left(t_{1}\right)
\end{array}\right| \cdot\left|\begin{array}{ll}
x_{\mu_{1}}\left(t_{2}\right) & x_{\mu_{1}}^{\prime}\left(t_{2}\right) \\
x_{v_{2}}\left(t_{2}\right) & x_{v_{1}}^{\prime}\left(t_{2}\right)
\end{array}\right| \cdots\left|\begin{array}{ll}
x_{\mu_{n}}\left(t_{n}\right) & x_{\mu_{n}}^{\prime}\left(t_{n}\right) \\
x_{v_{n}}\left(t_{n}\right) & x_{v_{n}}^{\prime}\left(t_{n}\right)
\end{array}\right|
$$

which after integration becomes

$$
2^{n} \sum \pm A\left(\mu_{1}, \nu_{1}\right) A\left(\mu_{2}, v_{2}\right) \ldots A\left(\mu_{n}, \nu_{n}\right),
$$


where $A(i, j)$ represents the area of the projection of $C$ on the plane $x_{i} 0 x_{j}$, while

$$
\mu_{1}, v_{1}, \mu_{2}, v_{2}, \ldots, \mu_{n}, v_{n}
$$

is an arrangement of the set of numbers $1,2, \ldots, 2 n$ into pairs such that $\mu_{i}<\nu_{i}$. It is clear that those arrangements (2.3) which differ only in the order of their pairs will contribute identical terms to the sum (2.2), there being $n$ ! such. Thus (2.2) reduces to

$$
2^{n} n ! \sum \pm A\left(\mu_{1}, v_{1}\right) \ldots A\left(\mu_{n}, v_{n}\right)
$$

where we sum only over such arrangements (2.3) in which the $n$ pairs are arranged lexicographically: $\mu_{1}<\mu_{2}<\cdots<\mu_{n}$. The sign of each term of $(2.4)$ is $(-1)^{I}$, where $I$ is the number of inversions in the permutation

$$
\left(\begin{array}{ccccc}
1 & 2 & \ldots & 2 n-1 & 2 n \\
\mu_{1} & \nu_{1} & \ldots & \mu_{n} & v_{n}
\end{array}\right)
$$

The number of terms in (2.4) is obtained by first counting pairs indiscriminately and then dividing by $n$ !, to throw out their order. We thus find in (2.4)

$$
\left(\begin{array}{c}
2 n \\
2
\end{array}\right)\left(\begin{array}{c}
2 n-2 \\
2
\end{array}\right) \cdots\left(\begin{array}{l}
4 \\
2
\end{array}\right)\left(\begin{array}{l}
2 \\
2
\end{array}\right) / n !=\frac{(2 n) !}{2^{n} n !}=1 \cdot 3 \cdot 5 \ldots(2 n-1)
$$

terms. On replacing the integral in (2.1) by its equivalent expression (2.4) we obtain the following

Theorem 2. If the closed curve $C$ is positively convex on $E_{2 n}$ then

$$
V(K(C))=\frac{2^{n}}{(2 n) !} \sum(-1)^{I} A\left(\mu_{1}, v_{1}\right) A\left(\mu_{2}, v_{2}\right) \ldots A\left(\mu_{n}, v_{n}\right)
$$

where $A(i, j)$ denotes the area of the projection of $C$ on the plane $x_{i} 0 x_{j}$, while the summation runs over all $1 \cdot 3 \cdot 5 \ldots(2 n-1)$ permutations $\mu_{1}, v_{1}, \mu_{2}, v_{2}, \ldots, \mu_{n}, v_{n}$, of the numbers 1, 2, ., $2 n$, such that $\mu_{1}<\nu_{i}$ and $\mu_{1}<\mu_{2}<\cdots<\mu_{n}$. Finally the exponent I is the number of inversions in the corresponding permutation.

Examples. 1. For $n=2$, (2.5) becomes

$$
V(K(C))=\frac{1}{6}\{A(1,2) A(3,4)-A(1,3) A(2,4)+A(1,4) A(2,3)\} .
$$

2. Let us evaluate $V\left(K\left(C_{0}\right)\right)$ for the curve $C_{0}$ defined by the equations (2) of our Introduction. For this case we find on inspection that

$$
A(\mu, v)=\int_{0}^{2 \pi} x_{\mu}(t) x_{\nu}^{\prime}(t) d t=0, \quad(\mu<v),
$$


unless $\mu$ is odd and $\nu=\mu+1$, when

$$
A(1,2)=\pi, A(3,4)=\frac{\pi}{2}, \ldots, A(2 n-1,2 n)=\frac{\pi}{n} .
$$

The sum (2.5) reduces now to one term only

$$
V\left(K\left(C_{0}\right)\right)=\frac{2^{n}}{(2 n) !} A(1,2) A(3,4) \ldots A(2 n-1,2 n)=\frac{2^{n} \pi^{n}}{n !(2 n) !} .
$$

Also the length of $C_{0}$ is easily found from (2):

$$
L=\int_{0}^{2 \pi} \sqrt{\sum x_{i}^{\prime 2}} d t=2 \pi \sqrt{n} .
$$

These values are seen to verify the isoperimetric relation (3) with the equality sign holding.

10. Reformulation of Theorem II in terms of Fourier series. We return to Theorem II of the Introduction and expand $x_{i}(t)$ in its Fourier series

$$
x_{i}(t) \sim \sum_{v=1}^{\infty}\left(a_{i v} \frac{\cos v t}{v}+b_{i v} \frac{\sin v t}{v}\right)
$$

where we assume the constant term to vanish without loss of generality. But then

$$
x_{i}^{\prime}(t) \sim \sum_{\nu=1}^{\infty}\left(-a_{i v} \sin v t+b_{i v} \cos \nu t\right),
$$

and Parseval's relation gives

$$
\int_{0}^{2 \pi} x_{i}^{2} d t=\pi \sum_{\nu=1}^{\infty}\left(a_{i \nu}^{2}+b_{i \nu}^{2}\right)
$$

whence

$$
\int_{0}^{2 \pi}\left(\sum_{1}^{2 n} x_{i}^{\prime 2}\right) d t=\pi \sum_{i=1}^{2 n} \sum_{\nu=1}^{\infty}\left(a_{i v}^{2}+b_{i v}^{2}\right)
$$

We also wish to express the right-hand side of (4) in terms of the Fourier coefficients $a_{i v}, b_{i v}$. This can be done in two ways leading to formally different but necessarily equivalent expressions.

A. We wish to show that the integral

$$
J=\int_{0}^{2 \pi} \ldots \int_{0}^{2 \pi} \operatorname{det}\left\|x_{i}\left(t_{1}\right), x_{i}^{\prime}\left(t_{1}\right), \ldots, x_{i}\left(t_{n}\right), x_{i}^{\prime}\left(t_{n}\right)\right\| d t_{1} \ldots d t_{n}
$$


may be expressed as

$$
J=(2 \pi)^{n} n ! \sum_{j_{1}<\cdots<j_{n}} \frac{1}{j_{1} j_{2} \ldots j_{n}} D\left(j_{1}, j_{2}, \ldots, j_{n}\right),
$$

where

$$
D\left(j_{1}, j_{2}, \ldots, j_{n}\right)=\operatorname{det}\left\|a_{i j_{1}}, b_{i j_{1}}, a_{i j_{2}}, b_{i j_{2}}, \ldots, a_{i j_{n}}, b_{i j_{n}}\right\| \text {. }
$$

Formally, there is no difficulty whatsoever. Indeed, if we introduce the expansions (2.6) and (2.7) into (2.9), writing the determinant as a sum of determinants obtained from the individual terms of the Fourier series, on using the orthogonality properties of the trigonometric system we find the expansion (2.10). A proof of the validity of (2.10), which includes a proof of the absolute convergence of the $n$-fold series (2.10), follows from the following remark: The integral $J$ is essentially the constant term of the $n$-fold Fourier expansion of the determinant under the integral sign. As we operate throughout within the class $L_{2}$, we will actually obtain that constant term by introducing formally the Fourier expansion of each element and gathering all terms which contribute to it. This, however, is precisely what was done above.

In terms of the quantities $S$ and $\Phi$, defined by (7) and (8), and using (2.8) and (2.10), the inequality (4) becomes

$$
\pi^{n} S^{n} \geq(2 \pi n)^{n} n ! \Phi,
$$

which is equivalent to (9). The equivalence of the Theorems II and III is now apparent if we refer to the Riesz-Fischer theorem.

B. An alternative expression for $J$, or equivalently for

$$
\Phi=\frac{1}{(2 \pi)^{n} n !} J
$$

may now be derived by observing the following: In Theorem 2 we have expressed $V(K(C))$ in terms of the $A(i, j)$ by the formula (2.5). It is clear that this result amounts to a similar expression for the integral $J$ without any reference to curves convex on $E_{2 n}$. In fact, by $(2.1)$ and $(2.5)$ we find

$$
J=2^{n} n ! \sum(-1)^{n} A\left(\mu_{1}, \nu_{1}\right) \ldots A\left(\mu_{n}, \nu_{n}\right),
$$

while Parseval's relation gives

$$
A(i, j)=\int_{0}^{2 \pi} x_{i} x_{j}^{\prime} d t=\pi \sum_{k=1}^{\infty} \frac{1}{k}\left(a_{i k} b_{j k}-a_{j k} b_{i k}\right) .
$$

Introducing now the new expressions 


$$
(i, j)=\sum_{k=1}^{\infty} \frac{1}{k}\left(a_{i k} b_{j k}-a_{j k} b_{i k}\right)
$$

we find, on combining the last four relations, the desired expression

$$
\Phi=\sum(-1)^{I}\left(\mu_{1}, \nu_{1}\right)\left(\mu_{2}, v_{2}\right) \ldots\left(\mu_{n}, v_{n}\right),
$$

which will be found useful in the next section.

\section{§ 3. A proof of Theorem III}

11. A first special case of Theorem III. Let us first establish the following Lemma 6. Let

$$
D=\operatorname{det}\left\|c_{i}\right\|
$$

be a real determinant of order $m$. Then

$$
\left(\sum_{i, j=1}^{m} c_{i j}^{2}\right)^{m} \geqq m^{m} D^{2}
$$

with the equality sign if and only if the elements of $D$ are proportional to the elements of an orthogonal matrix.

Proof. By Hadamard's inequality ${ }^{11}$ we have

$$
D^{2} \leq\left(\sum_{j} c_{1 j}^{1}\right)\left(\sum_{j} c_{2 j}^{2}\right) \cdots\left(\sum_{j} c_{m j}^{2}\right)
$$

By the inequality between the arithmetic and geometric mean we have

$$
\left(\sum_{i} c_{1 j}^{2}\right) \cdots\left(\sum_{j} c_{m j}^{2}\right) \leq\left(\begin{array}{l}
1 \\
m \\
\sum_{i, j} c_{i j}^{2}
\end{array}\right)^{m} \text {. }
$$

Notice that (3.3) and (3.4) imply (3.2). Moreover, equality in (3.2) implies the equality signs in both (3.3) and (3.4). Equality in (3.3) shows that the rows of $D$ are orthogonal to each other and the equality in (3.4) requires that

$$
\sum_{j} c_{1 j}^{2}=\sum_{j} c_{2 j}^{2}=\cdots=\sum_{j} c_{m j}^{2}
$$

which concludes a proof of Lemma 6 .

The special case of Theorem III which we have in mind is the case when all columns of the matrix $M$ vanish except the first $2 n$ columns. The inequality (9) reduces then to

1 See [6]. 


$$
\left\{\sum_{i=1}^{2 n} \sum_{\nu=1}^{n}\left(a_{i v}^{2}+b_{i \nu}^{2}\right)\right\}^{n} \geq(2 n)^{n} D(1,2, \ldots, n) .
$$

However, this inequality is implied by (3.2) for $m=2 n$ and $\left\|c_{i j}\right\|=M$. Moreover, the equality sign in (3.5) does not only imply, by Lemma 6 , that the elements of $M$ are proportional to those of an orthogonal matrix, but even that they are positively proportional to a right-orthogonal matrix. This establishes Theorem III in this special case.

12. An algebraic lemma. Let

$$
\Sigma=\left\|\sigma_{i j}\right\|_{i, j=1, \ldots, 2 n}
$$

be a real skew-symmetric matrix, which means that $\Sigma^{\prime}=-\Sigma$. We first establish the following identity in $x$ :

$$
\left|\begin{array}{cc}
-x I & \Sigma \\
\Sigma^{\prime} & -x I
\end{array}\right|=\left(P_{n}\left(x^{2}\right)\right)^{2}
$$

where $P_{n}(z)$ is a real polynomial of degree $n$.

Proof. By matrix multiplication we find

$$
\left\|\begin{array}{cc}
I & 0 \\
\Sigma^{\prime} & x I
\end{array}\right\| \cdot\left\|\begin{array}{cc}
-x I & \Sigma \\
\Sigma^{\prime} & -x I
\end{array}\right\|=\| \begin{array}{cc}
-x I & \Sigma \\
0 & \Sigma^{\prime} \Sigma-x^{2} I
\end{array} \mid
$$

and passing to determinants we have

$$
x^{2 n}\left|\begin{array}{cc}
-x I & \Sigma \\
\Sigma^{\prime} & -x I
\end{array}\right|=(-x)^{2 n}\left|\Sigma^{\prime} \Sigma-x^{2} I\right|
$$

whence the identity in $x$

$$
\left|\begin{array}{cc}
-x I & \Sigma \\
\Sigma^{\prime} & -x I
\end{array}\right|=\left|\Sigma^{\prime} \Sigma-x^{2} I\right|
$$

The right-hand side of this identity may now be factored. Indeed, by the skewsymmetry of $\Sigma$ we have

whence

$$
\left(\Sigma^{\prime}-x I\right)(\Sigma-x I)=\Sigma^{\prime} \Sigma-x\left(\Sigma+\Sigma^{\prime}\right)+x^{2} I=\Sigma \Sigma^{\prime}+x^{2} I
$$

$$
\left|\Sigma^{\prime} \Sigma+x^{2} I\right|=|\Sigma-x I|^{2}
$$

On the other hand

$$
\phi(x) \equiv|\Sigma-x I|
$$

$11-533807$. Acta mathematica. 91. Imprimé le 27 octobre 1954 . 
is an even polynomial in $x$, because

Thus

$$
\phi(-x)=|\Sigma+x I|=|-\Sigma-x I|=\left|\Sigma^{\prime}-x I\right|=|\Sigma-x I|=\phi(x) .
$$

and by (3.9) we have

$$
|\Sigma-x I|=P_{n}\left(-x^{2}\right) \text {, }
$$

$$
\left|\Sigma^{\prime} \Sigma+x^{2} I\right|=\left(P_{n}\left(-x^{2}\right)\right)^{2}
$$

Replacing $x^{2}$ by $-x^{2}$ we now obtain, via (3.8), the identity (3.7) which we wished to establish.

We may now state our

Lemma 7. If $\Sigma$ is a real skew-symmetric matrix of order $2 n$, then the symmetric matrix

$$
\Omega=\left\|\begin{array}{cc}
0 & \Sigma \\
\Sigma^{\prime} & 0
\end{array}\right\|,
$$

of order $4 n$, has at most $n$ distinct and positive characteristic values.

Indeed, the identity (3.7) gives precise information on the characteristic values of the matrix (3.10). It shows that they are all of multiplicity 2 and in pairs symmetric with respect to the origin, from which the conclusion of Lemma 7 follows.

13. A second special case of Theorem III: The matrix $M$ has only a finite number of non-zero columns. We assume throughout this Article 13 that

$$
a_{i j}=b_{i j}=0, \quad(i=1, \ldots, 2 n ; j=m+1, m+2, \ldots),
$$

where $m$ is a fixed number $>n$. Let us normalize the problem by requiring that

$$
S \equiv \sum_{\nu=1}^{m} \sum_{i=1}^{2 n}\left(a_{i v}^{2}+b_{i v}^{2}\right)=2 n .
$$

We are to show, then, that

$$
\Phi \leq \frac{1}{n !}
$$

with equality only under conditions as stated in Theorem III. This will be shown by maximizing the function $\Phi=\Phi\left(a_{i v}, b_{i v}\right)$ subject to the relation (3.11), a procedure which requires the first partial derivatives of the function $\Phi$. These derivatives are obtained as follows:

Let us denote by $\sigma_{i j}(i<j)$ the sum of those terms of the expression (2.13) which contain $(i, j)$ as one of their $n$ factors, with that factor $\left(\mu_{s}, v_{s}\right)=(i, j)$ removed. Let $i$ be fixed $(=1, \ldots, 2 n)$. On replacing the removed factors, (2.13) may now be written as 


$$
\Phi=\sum_{j=1}^{i-1}(j, i) \sigma_{j i}+\sum_{j=i+1}^{2 n}(i, j) \sigma_{i j}
$$

Indeed, this way of writing $\Phi$ represents a classification of the original terms of the sum (2.13) where all terms having $(j, i)$ as a factor $(j<i)$ form the class $(j, i) \sigma_{j i}$, and all terms having $(i, j)$ as a factor $(i<j)$ form the class $(i, j) \sigma_{i j}$. Thus in the case when $n=2$

$$
\Phi=(1,2)(3,4)-(1,3)(2,4)+(1,4)(2,3)
$$

if $i=3$, the classification (3.13) amounts to writing

where

$$
\Phi=(1,3) \sigma_{13}+(2,3) \sigma_{23}+(3,4) \sigma_{34}
$$

$$
\sigma_{13}=-(2,4), \quad \sigma_{23}=(1,4), \quad \sigma_{34}=(1,2) .
$$

We may also write (3.13) as

$$
\Phi=\sum_{j=1}^{i-1}(i, j)\left(-\sigma_{j i}\right)+\sum_{j=i+1}^{2 n}(i, j) \sigma_{i j} .
$$

If we extend the meaning of the symbol $\sigma_{i j}$ by agreeing that it be skew-symmetric in its subscripts, we may write the last relation as

$$
\Phi=\sum_{j=1}^{2 n}(i, j) \sigma_{i j}, \quad(i=1, \ldots, 2 n) .
$$

However,

$$
(i, j)=\sum_{k=1}^{m} \frac{1}{k}\left(a_{i k} b_{j k}-a_{j k} b_{i k}\right)
$$

and $\sigma_{i j}$ is independent (for all $j$ ) of the variables $a_{t k}, b_{t k}(k=1, \ldots, m)$, by its construction. From the last two relations we obtain the partial derivatives

$$
\frac{\partial \Phi}{\partial a_{i k}}=\sum_{j=1}^{2 n} \sigma_{i j} \frac{1}{k} b_{j k}, \frac{\partial \Phi}{\partial b_{i k}}=-\sum_{j=1}^{2 n} \sigma_{i j} \frac{1}{k} a_{j k} .
$$

Let us now assume that the elements of the matrix $M$ are such as to make $\Phi$ assume its absolutely maximal value subject to the condition $(3.11)$, or $S^{n}=(2 n)^{n}$. The Lagrange multiplier rule assures us of the existence of two constants $\mu$ and $\lambda$, not both vanishing, such that all first partial derivatives of the function

$$
G \equiv \mu \Phi-\lambda S^{n}
$$

vanish. ${ }^{12}$ We therefore have the $4 n m$ equations

12 See Carathéodory's book [3] for the formulation of the multiplier rule in the form needed here. 


$$
\begin{gathered}
k \frac{\partial G}{\partial a_{i k}} \equiv \mu \sum_{j} \sigma_{i j} b_{j k}-2 n \lambda k S^{n-1} a_{i k}=0, \\
k \frac{\partial G}{\partial b_{i k}} \equiv-\mu \sum_{j} \sigma_{i j} a_{j k}-2 n \lambda k S^{n-1} b_{i k}=0, \\
(i=1, \ldots, 2 n ; k=1, \ldots, m) .
\end{gathered}
$$

If we multiply these equations by $a_{i k} / k$ and $b_{i k} / k$, respectively, and add them all together, we obtain, in view of (3.15) and (3.14):

or

$$
0=\mu \sum_{i, j} \sigma_{i j}(i, j)-2 n \lambda S^{n}=2 n \mu \Phi-2 n \lambda S^{n}
$$

$$
\mu \Phi=\lambda S^{n} .
$$

However, the maximal value $\Phi$ is clearly positive (because $m>n$ ) and so is $S=2 n$. We conclude that both constants $\mu, \lambda$ are non-vanishing and we may therefore assume that $\mu=1$ and hence that $\lambda$ is positive.

The equations (3.16), with $\mu=1$, may be interpreted as follows: In terms of the matrices

$$
\Sigma=\left\|\sigma_{i j}\right\|_{1,2 n}, \quad \Omega=\left\|\begin{array}{cc}
0 & \Sigma \\
\Sigma^{\prime} & 0
\end{array}\right\|
$$

and the column vectors

$$
v_{k}=\left(a_{1 k}, \ldots, a_{2 n, k}, b_{1 k}, \ldots, b_{2 n, k}\right), \quad(k=1, \ldots, m),
$$

the $4 \mathrm{~nm}$ equations (3.16) may be written as $m$ matrix relations

$$
\Omega v_{k}=(2 n)^{n} k \lambda v_{k}, \quad(k=1, \ldots, m) .
$$

We have therefore reached the following conclusion: If the elements of $M$ maximize $\Phi$ subject to the condition (3.11), then there exists a positive constant $\lambda$ satisfying the relations $(3.20)$.

These relations show that if $v_{k} \neq 0$, then $(2 n)^{n} k \lambda$ is a characteristic value of the matrix $\Omega$. By Lemma 7 we know that $\Omega$ can have at most $n$ distinct positive characteristic values. We conclude that at most $n$ among the vectors (3.19) are nonvanishing. Let these be among the $n$ vectors $v_{j_{1}}, \ldots, v_{j_{n}}\left(1 \leq j_{1}<\cdots<j_{n} \leq m\right)$. But then, the expansion (8) reduces to a single term:

$$
\Phi=\frac{1}{j_{1} \ldots j_{n}} D\left(j_{1}, \ldots, j_{n}\right) .
$$

Since $\Phi>0$, we conclude that none of the vectors $v_{v_{1}}, \ldots, v_{j_{n}}$ vanishes. 
Now the solution of our problem is clear: 1 . If $j_{1}=1, \ldots, j_{n}=n$, then our pro. blem has already been solved (Article 11). 2. If $j_{n}>n$, then by the inequality (3.5) applied to $D\left(j_{1}, \ldots, j_{n}\right)$ we find

$$
n ! \Phi=\frac{n !}{j_{1} \ldots j_{n}} D\left(j_{1}, \ldots, j_{n}\right)<D\left(j_{1}, \ldots, j_{n}\right) \leq \frac{S^{n}}{(2 n)^{n}}=1
$$

whence $\Phi<1 / n !$, in contradiction to the fact that the maximal value of $\Phi$ should be at least $1 / n !$, by the result in the special case discussed in Article 11. This completes a proof of Theorem III for a "finite" matrix $M$, or if we wish, a proof of Theorem II for the case when the $x_{i}(t)$ are trigonometric polynomials.

14. Completing a proof of Theorem III. Let us now drop the assumption of a finite matrix $M$. Let again

$$
S=2 n
$$

hold and let us show that

$$
S^{n} \geq(2 n)^{n} n ! \Phi
$$

always holds, with the equality sign only as stated in Theorem III.

1. We can never have the reverse inequality

$$
S^{n}<(2 n)^{n} n ! \Phi
$$

Indeed, if this inequality were correct, let us truncate the matrix $M$ by replacing all columns beyond the $2 m$ th one by zero columns. For $m$ sufficiently large, the reverse inequality would still hold, in contradiction with the finite case already settled.

2. We can have the equality sign in (3.22) only in the case described by Theorem III. Indeed, let us assume that

$$
S^{n}=(2 n)^{n} n ! \Phi
$$

We may clearly assume $M$ to have infinitely many non-zero columns. We now proceed as follows: Let $m$ be a fixed number $>2 n$ and let us "unfreeze" the first $2 m$ columns of the matrix $M$ in such a way that (3.2I) is preserved. To these $4 m n$ variables we apply the multiplier rule as above, there being no difference from the previous case except that the finite sums (3.15) now become infinite series. Everything is the same as in Article 13, with the exception of the relation (3.17) which now becomes

where

$$
\mu \Phi_{m}=\lambda S^{n-1} S_{m}
$$




$$
\begin{aligned}
\Phi_{m} & =\sum_{i, j=1}^{2 n} \sigma_{i j} \sum_{k=1}^{m} \frac{1}{k}\left(a_{i k} b_{j k}-a_{j k} b_{i k}\right), \\
S_{m} & =\sum_{k=1}^{m} \sum_{i=1}^{2 n}\left(a_{i k}^{2}+b_{i k}^{2}\right) .
\end{aligned}
$$

However, since $\Phi_{m} \rightarrow \Phi$ and $S_{m} \rightarrow S$, as $m \rightarrow \infty$, it is clear that on choosing $m$ sufficiently large, we have $\Phi_{m}>0, S_{m}>0$, hence again $\mu=1, \lambda>0$. But then we find, as before, that at most $n$ among the vectors (3.19) may be non-zero. For sufficiently large $m$, this conclusion contradicts our assumption that $M$ has infinitely many nonzero columns.

\section{References}

[1]. T. Bonnesen and W. Fenchel, Theorie der konvexen Körper, Ergebnisse der Mathematik, 3, Heft 1 (1934).

[2]. C. Carathéodory, Über den Variabilitätsbereich der Fourierschen Konstanten von positiven harmonisehen Funktionen, Rendiconti di Palermo, 32 (1911), 193-217.

[3]. ---, Variationsrechnung und partielle Differentialgleichungen erster Ordnung, Leipzig, 1935.

[4]. F. GAN'TAKHER and M. KREIN, Oscillatory matrices and kernels and small vibrations of mechanical systems (in Russian), 2nd Hil., Moscow, 1950.

[5]. W. Gustrin, Sets of finite planar order, Juke Math. Journal, 14 (1947), $51-66$.

[6]. J. Hadamand, Résolution d'une question relative aux déterminants, Bulletin des sciences mathématiques, (2), 17 (1893), 240)-248.

[7]. J. Huetmstev, Introduction à la théorio des suites monotones, Oversigt over det $\mathrm{Kgl}$. Danske Videnskabernes Selskabs Forhandlinger, 1914, 1-74.

[8]. A. Hukwrq, Sur Ie problème des isupérimetres, Comptes Rendus, 132 (1901), 401-403; also in Math. Werke I, 490-49].

[9]. A. Marchaud, Sur les continus d'ordre borné, Acta Mathematica, 55 (1930), 67-115.

[10]. J. von Neumann and I. J. Schoenberg, Fourier integrals and metric geometry, Transactions of the Amer. Math. Society, 50 (1941), 226-251.

[11]. G. Pólya and G. Szeqö, łufgaben und Lehrsätze aus der Analysis, II, Berlin, 1925.

[12]. G. Pólya, On the mean-value theorem corresponding to a given linear homogeneous differential equation, Transactions of the Amer. Math. Society, 24 (1922), 312-324.

[13]. Peter Scherk, Über differenzierbare Kurven und Bögen, Časopis pro pěstováni Mate. matiky a Fysiky, 66 (1937), 165 191.

[14]. I. J. Schoenberg and ANNe Whitney, A theorem on polygons in $n$ dimensions with applications to variation-diminishing and cyclic variation-diminishing linear transformations, Compositio Mathematica, 9 (1951), 141-160.

[15]. I. J. SсноемberG, On smoothing operations and their generating functions, Bulletin of the Amer. Math. Society, 59 (1953), 199-230. 